\title{
Cancer Biomarkers Research Group
}

National Cancer Institute

\section{Source}

National Cancer Institute. Cancer Biomarkers Research Group. NCI Thesaurus. Code C16181.

The mission of the CBRG is to eng age basic and clinical scientists as well as epidemiologists and statisticians in a search for and validation of promising early cancer biomarkers. To do so, the CBRG supports and facilitates a broad spectrum of national and international research activities in molecular biology and genetics, particularly for the discovery of biomarkers for risk prediction and early detection of cancer. The CBRG also supports the development of databases and informatics systems to optimize tracking and assessment of biomarker utility and expression patterns. 